\section{Questions} vives

\section{Questions Vives}

Recherches en éducation

Vol. $8 n^{\circ} 15$ | 2011

Égalité filles-garçons : où en est-on?

\title{
Egalité filles-garçons : un objectif à l'épreuve du double usage des dispositifs d'accompagnement à la scolarité
}

Girl-boy equality: an objective against specific help project's dual-use

\section{Cyrille Morin}

\section{(2) OpenEdition}

\section{Journals}

\section{Édition électronique}

URL : http://journals.openedition.org/questionsvives/795

DOI : $10.4000 /$ questionsvives.795

ISBN : 978-2-8218-1090-7

ISSN : 1775-433X

\section{Éditeur}

Université Aix-Marseille (AMU)

\section{Édition imprimée}

Date de publication : 15 septembre 2011

ISBN : 978-2-912643-39-1

ISSN : 1635-4079

\section{Référence électronique}

Cyrille Morin, «Egalité filles-garçons : un objectif à l'épreuve du double usage des dispositifs d'accompagnement à la scolarité », Questions Vives [En ligne], Vol.8 n¹5 | 2011, mis en ligne le 10 octobre 2011, consulté le 19 avril 2019. URL : http://journals.openedition.org/questionsvives/795 ; DOI : 10.4000/questionsvives.795

Ce document a été généré automatiquement le 19 avril 2019.

\section{(†)

Questions Vives est mis à disposition selon les termes de la licence Creative Commons Attribution Pas d'Utilisation Commerciale - Pas de Modification 4.0 International. 


\title{
Egalité filles-garçons : un objectif à l'épreuve du double usage des dispositifs d'accompagnement à la scolarité
}

Girl-boy equality: an objective against specific help project's dual-use

\author{
Cyrille Morin
}

\section{Introduction}

1 Depuis le début des années 80 et la montée du chômage, de nombreuses initiatives ont vu le jour sur le territoire français pour aider les jeunes des catégories sociales les moins favorisées qui sont plus touchés car souvent moins qualifiés. "Aide aux devoirs", "soutien scolaire", "accompagnement scolaire» ou encore "accompagnement éducatif ", nombreuses sont les appellations désignant les dispositifs qui souhaitent prendre en charge, hors de l'école et du temps scolaire, ces enfants que l'on dit en «difficulté scolaire » et ceux qui pourraient l'être si on ne les aidait pas. Le travail de recherche sur lequel s'appuie cet article tente de comprendre comment et dans quels buts les enfants s'approprient ces dispositifs souvent associatifs ou proposés par des équipements municipaux de quartier. Nous appelons dispositifs d'accompagnement à la scolarité, ceux qui correspondent à la définition de la Charte de l'accompagnement scolaire (2001, p. 7) suivante :

On désigne par «accompagnement à la scolarité » l'ensemble des actions visant à offrir, aux côtés de l'Ecole, l'appui et les ressources dont les enfants ont besoin pour réussir à l'Ecole, appui qu'ils ne trouvent pas toujours dans leur environnement familial et social. Ces actions, qui ont lieu en dehors des temps de l'Ecole, sont centrées sur l'aide aux devoirs et les apports culturels nécessaires à la réussite scolaire. 
2 Nous ajouterons le terme «collectif» à cette nomination en lien avec notre terrain d'enquête ethnographique qui porte sur des accompagnements collectifs.

3 Les travaux de référence réalisés sur l'accompagnement à la scolarité ou d'autres dispositifs de remédiation, tels ceux de Glasman (2001), Piquée (2001) ou Bonnéry (2007), n'abordent que peu ou prou la question du genre des élèves fréquentant ces dispositifs. De même, les recherches portant sur les inégalités scolaires filles-garçons comme ceux de Baudelot et Establet (1992), ou sur la socialisation différenciée (Delalande, 2002 ; DafflonNovelle, 2006) n'abordent pas les difficultés scolaires d'apprentissage des filles et leur prise en charge par des dispositifs. Pourtant, des travaux sur l'échec scolaire (Duru-Bellat \& Marin, 2010) présentent le genre comme second facteur influant sur la scolarité après l'origine sociale des élèves. La question des inégalités de genre à l'école peut donc être posée à d'autres niveaux que ceux de la socialisation, de l'orientation ou du rapport au savoir. Reconnaître que l'échec scolaire frappe davantage les élèves défavorisé-e-s nous engage à réfléchir sur l'importance du facteur genre sur les trajectoires de ces élèves. Si effectivement les filles souffrent moins de cet échec, on peut tout de même se demander si l'objectif de lutte contre l'échec scolaire est atteint dès lors que ces dispositifs aident les enfants issu-e-s des classes sociales défavorisées, sans qu'ils tiennent compte des autres inégalités, notamment de genre, qui y sont déjà effectives. Il serait intéressant de chercher à voir si là aussi, on retrouve des inégalités filles-garçons.

4 L'accompagnement scolaire collectif se positionne entre l'école et la famille dans le quotidien des enfants, ce qui en fait un lieu singulier comme le souligne Glasman (2001). Il est fréquenté quotidiennement ou presque par les enfants dès la fin de la journée de classe et juste avant qu'ils et elles rentrent à leur domicile. C'est un « sas » entre l'école et la famille où l'on trouve, par la caractéristique " collective », une influence du groupe de pairs telle que présentée par Delalande (2002). La socialisation sexuée s'opère premièrement au sein de la famille (Belotti, 1975) puis par l'Ecole, qui participe à la construction sexuée du rapport au savoir (Mosconi, 2003). Dès lors, on peut se demander ce qu'il se joue dans l'accompagnement scolaire au niveau de la socialisation des enfants. En effet, les influences familiales, par la présence de la fratrie, scolaires par la nature de l'objet à travailler, et enfantines (par le groupe de pairs) se mêlent dans ce lieu interstitiel. Bien qu'il se veuille à l'écoute des besoins spécifiques des enfants, l'accompagnement scolaire collectif n'est-il pas un contexte où la socialisation sexuée va pouvoir se conforter et se développer de manière identique aux autres endroits fréquentés par les garçons et les filles? L'article se propose de montrer en quoi l'appropriation des dispositifs d'accompagnement scolaire par les enfants et l'encadrement réalisé par les adultes reflètent une socialisation socio-sexuée s'opérant à travers les «spécialisations » des dispositifs d'une part et par les rapports de genre entre acteurs, enfants et adultes d'autre part. Ainsi nous verrons, premièrement, comment l'organisation et les spécialisations des dispositifs peuvent véhiculer involontairement des représentations sexuées auprès des enfants. Puis, nous observerons en quoi l'accompagnement scolaire est le «théâtre» de l'apprentissage de compétences traditionnellement attribuées aux femmes. Ce passage nous permettra d'aborder également la question de l'orientation, dont les jeunes parlent volontiers avec les adultes encadrant-e-s. 


\section{Croisement de quelques recherches sur l'accompagnement à la scolarité, la socialisation sexuée, et le rapport au savoir}

Nous avons cité précédemment des recherches en indiquant le complément que nous souhaitions apporter. Notre réflexion s'appuie aussi sur les éléments qu'elles ont mis au jour. Ainsi, M. Duru-Bellat (2010) rappelle que les inégalités filles-garçons face à l'école s'estompent en fonction de leur catégorie sociale pour expliquer que les garçons sont plus touchés par l'échec scolaire. Elle indique également que les filles ne sont concernées que dans une moindre mesure par ce phénomène. Elles sont pourtant très présentes dans les dispositifs d'accompagnement, ce qui ne signifie pas qu'elles soient nécessairement en difficulté. En effet, les dispositifs d'accompagnement n'ont pas les difficultés d'apprentissage comme unique critère d'éligibilité et adoptent régulièrement la demande des parents et/ou des enfants comme critère révélant un besoin social aussi important que le besoin scolaire. Si l'on accepte l'idée de Baudelot et Establet (2008, p. 100) selon laquelle "les filles construisent aisément leur identité sexuée dans l'univers scolaire et son environnement culturel proche ", on comprend cette forte présence des jeunes filles qui " éduquées à l'attention à autrui ont moins de mal à anticiper les injonctions des adultes dans la société scolaire» (ibid. p. 97). Ainsi, leurs aptitudes à répondre aux attentes de l'école peuvent être mobilisées pour toute la question scolaire, y compris hors de l'école et dans les dispositifs d'accompagnement scolaire. Ceux-ci sont des aides face aux apprentissages et à la scolarité, et, tout comme l'école, ils apparaissent sous la forme d'un lieu familier et d'un moment de la journée. Le transfert des comportements scolaires dans ce lieu n'est donc pas surprenant.

Mais il y a un aspect fondamental que ne manque pas d'indiquer Glasman (2001): l'accompagnement scolaire se situe, dans l'idéal, à égale distance de la famille et de l'école. Si l'on remarque lors des séances d'accompagnement des traces du comportement demandé par l'école (calme et concentration par exemple) chez les enfants bénéficiaires, on remarque que ceux-ci et celles-ci s'en défont assez facilement au fil des minutes ou de l'achèvement du travail scolaire pour laisser place au comportement accepté par le groupe de pairs et par la famille. Dans ce sens, on voit, durant chaque séance, les élèves s'extirper de l'influence de l'école pour suivre celle du groupe d'enfants ou de la famille, et parfois même les deux. C'est ce qui nous amène à considérer les dispositifs d'accompagnement comme une sorte de carrefour entre les socialisations enfantine, familiale, et scolaire où chacune trouverait sa place d'une manière très visible bien que variable en fonction des étapes des séances. Nous ne cherchons pas à dire que ces différentes socialisations ne sont pas présentes à chaque instant, ce qui serait faux, mais que l'accompagnement scolaire est un moment qui, dans le quotidien de l'enfant, les met en présence distinctement et non de manière diffuse. En effet, tant qu'ils et elles font leurs devoirs, les enfants ont des attitudes proches de celles des élèves en classe (concentration, chuchotement, stratégies d'évitement des adultes pour faire des bêtises), mais dès qu'ils et elles ont fini leurs devoirs, ils et elles adoptent des comportements de détente hérités des socialisations familiales et enfantines (Delalande, 2002). Ces comportements, qui transparaissent par le biais des communications entre enfants, varient de registre plus ou moins respectueux selon qu'ils ou elles s'adressent à la grande sœur ou à leurs copains par exemple. 
7 Cependant, quelque soit le type de socialisation, il y a une distinction sexuée avec à chaque fois des conséquences sur la vie des individus. A l'école, le comportement des adultes encadrant-e-s, et notamment celui des enseignant-e-s envers les filles et les garçons, influe sur le rapport aux savoirs des élèves (Mosconi, 2003) et ultérieurement sur leur orientation (Duru-Bellat, 2008). C'est ainsi que les élèves sont préparé-e-s à certaines carrières plutôt qu'à d'autres en fonction de leur genre. De même, la famille et les ami-e-s contribuent à une socialisation sexuée dans la sphère privée et ce dès le plus jeune âge (Belotti, 1975). Cette socialisation indique aux enfants comment se comporter dans la vie privée et quels choix faire pour diriger leur vie conformément à ce qui est attendu en fonction du sexe biologique. Famille et ami-e-s encouragent ainsi des comportements traditionnellement attribués aux hommes ou aux femmes, à travers des choix de vêtements mais aussi de jeux et d'activités de loisirs qui vont inciter l'enfant et l'adolescent-e à correspondre à ce que l'on attend d'eux et d'elles à l'école, ainsi que dans les choix d'orientation et de construction de la vie adulte. Par exemple, l'anticipation de la maternité par les filles influe sur leur choix de carrière. Cette anticipation s'opère dans la logique de préparation au devoir parental présent dans l'éducation familiale des filles par le développement de capacités assimilables au care, terme renvoyant au travail de soin et de prise en charge d'autrui, comme par exemple les enfants, et ce que la réalisation des tâches soit rémunérée ou non (Bereni, Chauvin, Jaunait \& Revillard, 2008). Cet ensemble de compétences "naturellement » féminines doivent être acquises par les jeunes filles. Nous verrons que l'accompagnement à la scolarité peut être pour les jeunes filles un contexte à l'apprentissage simultané de ces capacités et du comportement face à leur orientation.

8 Toutefois, le choix d'une orientation par l'élève ne relève pas uniquement des inclinaisons de la socialisation familiale, mais aussi du rapport au savoir des individus. Ainsi, le dernier rapport Filles-Garçons : sur les chemins de l'égalité (2010) indique qu'à notes égales à celles des garçons en mathématiques, les jeunes filles ne se sentent pas aussi compétentes et s'orientent plus facilement vers des filières littéraires ou plus traditionnellement "féminines ». L'orientation des élèves est une réflexion qui engage bien évidemment les jeunes concerné-e-s mais aussi une implication des parents et de l'institution scolaire pour lesquel-le-s les conséquences de cette orientation ne sont pas sans importance. L'école propose des orientations possibles à l'enfant en fonction de ses résultats scolaires et d'indicateurs tels que son comportement et de ce qu'elle pense convenir le mieux. La famille fait des projections dans l'avenir pour ses enfants, valorisantes ou non, en fonction de l'éducation qu'elle a apportée et de ses représentations des normes de genre. Dans ce contexte de choix d'orientation où les influences simultanées du milieu familial et scolaire vont faire pression sur l'enfant quant à son orientation, quel peut-être le rôle de l'accompagnement à la scolarité pour les jeunes filles y adhérant? C'est une des questions que nous tenterons d'aborder en examinant les inégalités scolaires filles-garçons par le prisme des dispositifs d'accompagnement scolaire collectifs.

\section{Méthodologie : une approche ethnographique de l'accompagnement à la scolarité}

9 Cet article prend source dans une recherche en cours sur quatre dispositifs d'accompagnement à la scolarité en collectif, situés dans un quartier dit « populaire » et 
dont les établissements scolaires sont classés en ZEP. Nous appellerons les quatre dispositifs $A, B, C$ et $D$. Ils accueillent chacun de 20 à 25 enfants inscrit-e-s, dont 10 à 15 régulièrement. $C$ a fermé ses portes en mars 2010. Le recueil empirique englobe des observations participantes et des entretiens compréhensifs réalisés sur les années scolaires 2008 à 2010. Je me suis présenté en tant qu'étudiant et bénévole auprès des jeunes (de 6 à 18 ans). Cette posture était facilitée par ma proximité d'âge avec celui des nombreus-e-s bénévoles étudiant-e-s. Quelques bénévoles étaient des retraité-e-s issu-e-s de l'enseignement ou de métiers de la formation professionnelle. J'ai présenté l'enquête comme un travail d'étude universitaire sans en préciser le niveau, pensant que le terme «doctorat » pouvait être très fortement connoté positivement ou négativement. Cette précaution vient du fait, également relaté par Beaud et Weber (2008), que les habitants des quartiers dits "populaires" voire « sensibles" peuvent avoir des réactions de rejet liés au sentiment stigmatisant d'être pris pour des cobayes ou des «cas soc'" comme nous l'avons entendu sur notre terrain. Prendre le rôle d'étudiant bénévole a permis de minimiser d'éventuelles connotations négatives liées à la démarche de recherche. Je dois également indiquer que l'université de la ville dans laquelle j'enquête a mis en place un système de Validation de l'Engagement Etudiant (V.E.E.), qui demande aux étudiant-e-s bénévoles de faire un dossier pour lequel des entretiens et des questionnaires sont régulièrement passés avec les enfants bénéficiaires des dispositifs. La démarche de sollicitation pour des entretiens n'était donc pas inconnue à la plupart des jeunes interviewé-e-s. Les entretiens ont été faits sur le lieu des accompagnements après la réalisation des devoirs, comme cela nous a été demandé.

Nous avons interviewé des jeunes (35), filles (22) et garçons (13), issu-e-s du quartier et des immeubles proches des lieux d'accompagnement. La forte représentation des filles dans ces dispositifs est palpable à travers l'inégale répartition des entretiens selon le genre et nous incite à ne pas présenter la démonstration suivante sous forme d'analyse proprement comparative mais plutôt centrée, sans exclusivité, sur la situation des jeunes filles. Les jeunes qui adhèrent aux accompagnements par fratrie ont des âges allant de 6 ans (inscription en CP est le minimum obligatoire) à 18 ans (il y a trois lycéennes). Tous et toutes, sauf une des fillettes, parlent une deuxième langue à la maison, par choix familial ou parce que leurs parents ne sont pas francophones. Certain-e-s jeunes sont inscrit-e-s par fratrie dans plusieurs dispositifs, ce qui rend les chiffres cités précédemment encore plus approximatifs et expliquent le faible nombre d'entretiens par rapport au nombre d'inscrit-e-s. Ce phénomène de pluri-inscriptions est le point de départ de notre travail sur ces dispositifs dits « collectifs » car il nous semble révélateur d'un investissement des enfants et des familles envers la scolarité. Cet intérêt fort de la part des familles n'était prévu et anticipé ni par les dispositifs en question, ni par la politique éducative de la ville et les financeurs. Les places étant peu nombreuses pour le nombre d'enfants, les inscriptions par fratrie et famille élargie, souvent pendant plusieurs années, questionnent l'impact des dispositifs sur le quartier puisque finalement peu d'enfants sont bénéficiaires. La question du genre a fini par s'imposer ultérieurement, à la lecture des données obtenues par la méthode ethnographique.

11 Les données empiriques ont permis de dessiner un "portrait » des enfants bénéficiaires ainsi que des bénévoles et des référent-e-s. Nous avons rassemblé, pour chaque acteur et actrices, l'ensemble des données, que nous avions recueillies par entretien ainsi que par observation, via la rédaction de fiches biographiques, qui permettent de reconstituer le parcours d'adhésion aux dispositifs. Ces fiches nous dévoilent en fait les éléments de 
présentation des individus (âge, sexe, catégorie socioprofessionnelle de la personne responsable de famille,etc.) mais aussi l'ensemble des évènements auxquels ils et elles ont participé, ainsi que les éléments d'entretiens qui nous paraissent les plus significatifs pour comprendre le sens des interactions qu'ils et elles ont avec les autres individus. De cette manière, nous avons pu reconstituer les réseaux d'interrelations entre les différente-s acteurs et actrices, et mieux comprendre les phénomènes de déplacement et/ou de stagnation des groupes d'enfants d'un dispositif par rapport à un autre. Notre analyse de l'accompagnement à la scolarité se fait donc dans une approche interactionniste qui nous semble la plus pertinente pour apprécier les nuances des données recueillies. L'objectif de la recherche est d'établir un état des interrelations autour de la situation de chaque enfant ou fratrie de manière à comprendre en quoi l'accompagnement à la scolarité répond ou non à la demande de prise en charge des besoins qu'ils soient d'ordre scolaire ou social. Le projet de recherche était donc initialement assez éloigné des questions de genre qui ont néanmoins rapidement émergé.

\section{L'organisation des dispositifs : un véhicule pour les représentations sexuées?}

Nous avons récolté ces premières données en entrant en contact avec notre terrain d'enquête, c'est-à-dire en prenant rendez-vous avec les référent-e-s des dispositifs qui acceptaient de nous accueillir. Deux types d'éléments nous sont alors apparus. Premièrement, la population des référent-e-s est très fortement féminisée, quatre femmes professionnelles, un homme bénévole (enseignant de métier) et un professionnel soit les deux tiers et plus si l'on opère une distinction par le caractère professionnel de l'activité de référent-e. Cette surreprésentation des femmes n'est pas un fait surprenant et confirme la féminisation des métiers de l'animation déjà soulignée par Gillet et Raybaud (2006). Deuxièmement, les premiers contacts au sein des locaux d'accompagnement, en l'absence d'élèves, ont permis d'apercevoir des traces de vie du groupe (ex : jeux mal rangés, dessins éparpillés ou affichés) nous permettant de dire qu'il s'y passe plus de choses que la réalisation du travail scolaire.

13 Selon Glasman (2001), le local d'accompagnement scolaire est pensé par les référent-e-s comme un lieu à visée éducative mais aussi comme un espace intermédiaire à l'école et au domicile des enfants. L'organisation de l'espace en fonction des objectifs pédagogiques du projet atteste de fait de l'intention éducative du lieu. Toutefois, la notion d'espace intermédiaire entre l'institution scolaire et la famille peut être reconsidérée car l'enfant n'est pas seul avec un ou une bénévole lors des séances mais bien en présence d'un groupe composé de deux "sous-groupes" d'adultes et d'enfants. L'ensemble des individus concernés construisent un groupe dont la tonalité positive ou négative des interactions aux yeux de l'élève va faire qu'il ou elle adhère ou pas, soit assidu-eou absentéiste aux dispositifs. Le local d'accompagnement n'est finalement pas un intermédiaire uniquement entre les deux institutions que sont la famille et l'école, mais également avec le groupe de pairs et la société dans son ensemble à travers la présence de bénévoles n'étant pas issu-e-s du quartier.

14 Ce lieu est organisé, souvent par des professionnels et parfois par des bénévoles comme c'est le cas pour B. Mais quel que soit le statut des personnes référentes, ces locaux sont pensés de manière à optimiser l'accueil des jeunes, si possible en correspondant aux 
objectifs du projet pédagogique, bien que les contraintes logistiques et financières jouent souvent en leur défaveur. On peut d'ailleurs voir dans le dispositif $A$, l'influence de l'organisation du lieu. Lorsque nous demandons à la référente pourquoi un seul garçon fréquente le dispositif $A$, elle nous explique "Je ne sais pas... en fait je ne sais pas du tout... c'est vrai qu'il y a deux ou trois ans il y en avait encore qui venaient là tout un groupe mais depuis plus rien alors je sais pas pourquoi...mais je les ai vus y a quelques semaines à $C$ et je crois qu'ils $y$ vont parce qu'il y a un baby-foot là-bas donc je crois c'est pour cela qu'ils restent là bas c'est vrai que nous ici on n'a pas grand-chose pour les garçons». Ici le "manque » de matériel adapté serait la cause de l'abandon du lieu par les garçons, mais il existe d'autres facteurs importants expliquant la fréquentation des dispositifs (emplacement, lien avec les référent-e-s et les bénévoles par exemple).

L'emplacement géographique qu'ils occupent dans le quartier influe sur le quotidien de ces dispositifs. Ainsi, $A$ et $B$ sont situés dans des locaux en bas des tours d'habitations à loyers modérés, ce qui crée pour les enfants une proximité entre l'accompagnement et leur domicile souvent situé dans les étages au dessus ou de l'autre côté du square. $C$ et $D$ sont dans l'ancienne maison de quartier, l'un ayant récupéré les locaux de l'autre après sa fermeture. La proximité des accompagnements les uns par rapport aux autres est un facteur important pour comprendre l'appropriation qui en est faite par les jeunes. En effet, les filles aînées des fratries que nous avons pu rencontrer placent leurs frères et sœurs dans des dispositifs proches qui accueillent les élèves de primaire, tandis qu'elles se rendent à ceux acceptant les collégien-ne-s. Ainsi, la multiplicité des accompagnements accessibles permet aux jeunes filles de déposer leurs cadet-te-s au dispositif $A$ et de se rendre ensuite seule à $D$ qui n'accueille que des collègien.ne.s et est par ailleurs coordonné par une jeune femme. Le dispositif $B$, qui est ouvert à tous les élèves du collège n'est fréquenté que par des collégiens. C'est ce qui nous a amené à interroger non seulement l'emplacement des accompagnements mais également, l'organisation de ceuxci en fonction des "spécialisations » et des répartitions entre hommes et femmes aux postes d'encadrement et d'animation.

\section{Une division sexuée dans le travail de référent-e-s de dispositif ?}

16 Comme nous l'avons souligné plus haut, les femmes sont surreprésentées parmi les référent-e-s de dispositif. Présenter brièvement l'organisation de l'encadrement des dispositifs d'accompagnement à la scolarité nous paraît important pour comprendre comment ils sont conçus et nous permet de voir, à ce premier niveau, des éléments de la division sexuée du travail proche de ceux relevés par Jaboin (2010) chez les équipes pédagogiques de maternelles. Sur les quatre dispositifs que nous avons rencontrés, trois sont coordonnés par des femmes professionnelles, un par un bénévole et un par un homme professionnel. Les référentes gèrent l'accueil des enfants de primaire. $B$, qui est dirigé par un homme, n'accueille que des garçons lycéens et collégiens et uniquement pour un travail sur les sciences dures (mathématiques, physique, chimie...). $D$ accueille séparément les élèves de primaire, encadrés par deux référentes, et ceux du collège qui sont encadrés par un référent. $A$ et $C$ sont encadrés par des référentes mais pour ce dernier un espace jeunes, animé par un homme, a été ouvert juste à côté afin d'accueillir les jeunes bénéficiaires après leurs devoirs ainsi que les autres jeunes du quartier. 
17 La division déjà énoncée est marquée par deux points: les "spécialisations » par discipline et les tranches d'âges. Les disciplines scientifiques et les jeunes les plus âgés sont encadrés par des hommes. L'encadrement de collégien-ne-s par la référente de $C$ n'est effectif que lors du temps de réalisation des devoirs. Concernant $D$, deux femmes s'occupent des primaires, et c'est un homme qui encadre les adolescents. Au dispositif $A$, plus spécialisé dans le français et les langues, on trouve des élèves de primaire (CP, CE1 et $\mathrm{CE} 2$ ) et des collégiennes. Seul un collégien y vient et très rarement. Enfin, $B$ qui est spécialisé en mathématiques, physiques et chimie est ouvert aux collègien.ne.s mais n'est fréquenté que par des garçons.

18 A propos des spécialisations, les dispositifs $A$ et $B$, respectivement spécialisés en français et langues et le second en sciences, on remarque que $A$ est majoritairement fréquenté par des filles (un garçon), et que B n'accompagne que des garçons. Par ailleurs, la fréquentation de $A$ par des garçons de primaire est due à son ancienne ou actuelle fréquentation par leurs grandes sœurs qui y viennent également ou qui les y déposent. La participation majoritaire des garçons au dispositif $B$ se retrouve également dans l'accueil de collègien.ne.s du dispositif $D$, bénéficiant du bénévolat d'une enseignante de mathématiques mais qui n'accueille paradoxalement que trois garçons. Concernant la répartition des référent-e-s par tranche d'âge, on remarque que ce sont à chaque fois des femmes qui ont en charge les élèves les plus jeunes malgré la présence d'hommes (dispositifs $C$ et $D$ ).

On pourrait penser que cet état des lieux reflète une organisation volontairement sexuée des tâches. Cependant, il est plus probable qu'elle soit due à la forte féminisation des métiers de l'animation; ce qui correspond à une division sexuée des tâches appréhendable à l'échelle du marché du travail dans son ensemble. En 2002, $70 \%$ des professionnel-le-s de l'animation étaient des femmes (Bacou, 2006). Les données recueillies ne permettent pas d'affirmer que cette répartition genrée ait été réfléchie consciemment par les référent-e-s. Cela paraît peu envisageable tant ils et elles reconnaissent avoir peu de lien les un-e-s avec les autres. Cependant, les conséquences de cette organisation sont tout de même intéressantes car elles laissent apparaître une division sexuée, d'autant plus marquante qu'elle semble inconsciente, au niveau des répartitions par tranche d'âge, mais aussi au niveau des spécialisations des savoirs et savoir-faire.

En effet, les femmes sont affectées à la réalisation des devoirs et au travail scolaire et particulièrement avec les plus petits. Dans le dispositif $D$, c'est une femme qui s'occupe des devoirs des collégiens mais c'est avec un homme qu'ils se rendent aux activités de loisirs. Enfin, dans le dispositif $C$, nous avons constaté au fil des observations que le référent de la partie du dispositif qui accueille les collégiens n'est que très peu présent. Il m'explique :

Je laisse la place à la bénévole car il n'y a que trois jeunes et je ne suis pas loin.

De manière assez complexe, sans doute lié au caractère inconscient et non concerté de cette répartition des tâches, on retrouve des traces d'une hiérarchisation sexuée des tâches de travail. C'est un phénomène qu'Yveline Jaboin (2010) a également identifié chez les équipes pédagogiques mixtes de maternelle. Comme le fait remarquer cette auteure, la division, même involontaire, n'en reste pas moins perceptible par les enfants bénéficiaires, et l'on peut voir se profiler la question du rapport au savoir à travers les fréquentations genrées des dispositifs. 


\section{Parcours d'adhésion des enfants et rapport sexué aux savoirs}

mathématiques anciennement bénévole du dispositif $C$ et qui n'est présente que le mardi. Kévin n'est pas venu de son propre chef à l'accompagnement. Ce sont ses parents qui, par le bouche à oreille avec d'autres parents d'élèves de sa classe, ont entendu parler du dispositif. Kévin y retrouve des camarades du collège et y vient avec plaisir. Chaque soir, un de ses parents vient le chercher pour le reconduire chez lui ou bien la référente le raccompagne. Il en est de même pour Eric. Ses parents viennent le chercher et insistent pour qu'il vienne bien deux fois par semaine. Eric est turbulent en classe mais très calme à l'accompagnement. Il n'a pas non plus de difficultés particulières mais, comme pour Kevin, ses parents disent :

On n'est pas au niveau. On n'a pas eu les mêmes choses et c'est oublié...

Nous venons de voir que les offres d'accompagnement scolaire et leur organisation coïncident avec des répartitions singulières des filles et des garçons au sein de ces dispositifs. Mais cette répartition des bénéficiaires n'est pas si aléatoire qu'il y parait. En effet, Olivia, référente du dispositif $A$, dit savoir «à peu près qui est spécialisé dans quoi » et reconnaît des compétences diversifiées aux différents dispositifs présents sur le territoire. Elle nous explique également qu'elle "redirige » les élèves en fonction des besoins qu'ils ou elles expriment. On remarque ainsi que le dispositif ne s'adapte que dans une certaine marge aux élèves qui vont finalement devoir s'adapter et s'orienter face à l'offre d'accompagnement, comme ils le feront ou le font déjà avec les filières scolaires. Choisir un dispositif n'est donc plus anodin. On ne vient pas uniquement pour faire ses devoirs mais bien parce que l'on peut y solliciter certaines compétences de bénévoles. Ainsi Kévin, collégien de 12 ans, vient à $D$ et m'a expliqué dès le premier jour et sa première séance avec moi :

Je viens ici parce qu'on a dit à mon père que je pourrais travailler sur les maths le mardi et l'autre jour sur d'autres trucs, le français et tout.

4 Par ailleurs, le corpus de données fait ressortir une forte tendance des jeunes à penser que faire les devoirs à la maison est souvent source de conflits avec les parents. En ce sens, l'accompagnement scolaire répond à une demande sociale autant que scolaire. Pour ces deux garçons, le rapport au savoir n'est pas le moteur de leur venue à l'accompagnement. Il s'agit d'une décision parentale. Néanmoins, il arrive aussi fréquemment que les jeunes filles ou garçons soient «moins bons » ou « aiment moins » une discipline; ce qui les conduit parfois à de mauvais résultats et suscite de leur part des demandes spécifiques qu'ils ou elles expriment seul-e-s et spontanément aux référent-e-s. C'est un processus normal et explicable par l'étude de Safont-Mottay, Oubrayrie-Roussel et Prêteur (2010), qui montre que les garçons et les filles accordent la même valeur à l'école. C'est ce qui a conduit Elodie vers le dispositif $A$. Karine sa grande sœur, qui y vient depuis six ans, a demandé à la référente si quelqu'un pouvait venir aider sa sœur en français. Rappelons que ce dispositif est spécialisé en français et langues; ce qui explique que la référente n'ait pas eu de mal à entamer les démarches pour trouver une bénévole enseignante de français. Pour d'autres jeunes filles fréquentant ce dispositif la situation est similaire même si aucun bénévole n'est là spécifiquement pour elles. Les collégiennes et lycéennes y viennent lorsqu'elles ont des exercices ou des contrôles en anglais et en 
espagnol. Les compétences en français sont plutôt sollicitées par les frères et sœurs de primaire qui doivent apprendre la lecture et l'écriture. Parmi les élèves du dispositif $A$, on trouve également Thiphaine et Mathilde. Toutes les deux collégiennes, elles sont également cousines et inséparables. Elles arrivent en même temps à l'accompagnement et repartent, quand leurs frères et sœurs ne les sollicitent pas, en même temps. Elles se sont inscrites ensemble à l'accompagnement qu'elles fréquentent depuis six ans, époque de leur CM1. Elles y viennent pour travailler leurs devoirs et plus spécialement l'anglais, mais surtout parce que c'est "chez Olivia». C'est comme cela qu'elles appellent le dispositif ainsi que le font tous les enfants qui le connaissent. Cette assimilation de la référente au lieu d'accompagnement nous montre qu'il existe un facteur affectif très important concernant la fréquentation des dispositifs par les élèves. Leur assiduité n'étant pas imposée, parce que pas imposable, celle-ci est dépendante du lien de confiance et d'amitié souvent très fort qui se crée entre les enfants et les adultes encadrant-e-s. Thiphaine et Mathilde expliquent qu'elles aiment fréquenter le dispositif $A$ parce qu'il y a Olivia. D'un besoin en termes de suivi scolaire s'est ainsi créée une relation affective conditionnant le lien entre les adultes et les jeunes suivi-e-s.

Cependant, ce lien affectif entre adultes et enfants ne se fait pas systématiquement et l'on remarque que les filles, comme le font Tiphaine et sa cousine, ont une tendance à aller dans des accompagnements animés par des femmes et non par des hommes, mêmes quand ceux-ci se trouvent dans le même lieu. L'adhésion des jeunes filles semble aller plus facilement vers un dispositif dont l'offre disciplinaire leur plaît, à condition qu'il ait une référente. Les enfants viennent dans un dispositif par la réponse à la demande scolaire qu'il peut leur procurer, sans pour autant omettre l'aspect affectif lié aux affinités établies avec les adultes et les enfants.

En effet, la satisfaction d'ordre scolaire s'accompagne souvent de celle liée à l'opportunité de pouvoir fréquenter les copains et copines de classe après l'école, dans un espace prévu pour des activités scolaires mais aussi ludiques.

Parmi, l'ensemble des enfants rencontré-e-s, pas un-e n'est l'unique représentant-e de sa classe au sein du dispositif. Ce phénomène s'explique par la communication entre parents d'élèves mais aussi parfois par la présentation des dispositifs aux parents par les enseignants. Cependant, les adultes ne sont pas les seuls à opérer un pouvoir sur l'inscription aux dispositifs puisque les parents confient fréquemment la responsabilité des frères et sœurs aux jeunes filles pour le temps de l'accompagnement et des trajets pour y aller et en revenir.

\section{Accompagnement scolaire, développement du « care » et orientation scolaire des collégiennes}

28 L'accompagnement scolaire se déroule hors du temps scolaire, mais après l'école ne signifie pas qu'il s'agisse de temps libre pour les jeunes qui y viennent. Des jeunes filles collégiennes telles que Tiphaine, Mathilde ou encore Sandrine, que nous avons rencontrées lors de notre recherche expliquent qu'elles doivent «surveiller les petits». Nous avons pu remarquer à plusieurs reprises durant nos observations qu'elles s'interrompaient dans leurs devoirs pour arrêter une dispute entre les plus petits si un des membres de leur fratrie était concerné. Ce type d'évènement de surveillance des cadet-te-s sur leurs comportements vis-à-vis des adultes mais aussi avec les autres 
enfants se répète fréquemment mais à chaque fois elles interviennent avant que la situation soit prise en charge par les adultes. Lorsqu'il arrive que les plus jeunes, les "petits", n'écoutent pas elles préviennent «si tu m'écoutes pas tu verras ça avec ta mère!» [Tiphaine] ou d'autres formulations ayant pour but de montrer qu'ils et elles doivent les écouter car elles en sont responsables. Ce rôle n'est pas forcément réfléchi par elles bien qu'elles aient conscience d'être investies de responsabilités déléguées par leurs parents. Au détour d'une réponse en entretien nous abordons avec Mathilde la fermeture prochaine du dispositif A :

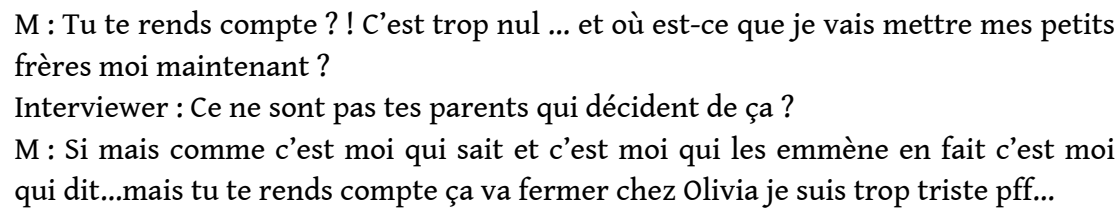

Les parents font confiance à la jeune fille pour s'occuper de ses frères et sœurs quand ils ne peuvent être là; ce qui implique qu'elle doit savoir s'en occuper eux comme ils l'auraient fait. Techniquement, l'ensemble des gestes qui ont pu être observés concernant l'aide et le soin à apporter aux plus petit-e-s ; et ceux que l'on rapproche des compétences du care sont les suivants : apport du goûter, dépôt à l'accompagnement et récupération, médiation lors des disputes avec d'autres enfants, soins et réconfort lors des «bobos » et des pleurs, veille au respect des adultes et à la politesse, vérification des devoirs et du bon comportement auprès des adultes si elles n'étaient pas présentes, rappels à l'ordre sous forme de demande de calme et de concentration sur les devoirs lorsqu'elles sont présentes, vérification de la tenue et du soin aux affaires. Il s'agit de tâches traditionnellement affectées aux parents et particulièrement aux mères. Ces jeunes filles apprennent à les effectuer en lien avec la fréquentation des dispositifs d'accompagnement à la scolarité. Les comportements de prise de responsabilités de la part des jeunes filles ne préoccupent pas les référent-e-s d'accompagnement alors que les garçons ne sont pas sollicités pour ce type de tâches. Nicolas, petit frère de Tiphaine avec deux ans de moins, est également au collège mais ne s'occupe pas de sa petite sœur qu'il renvoie systématiquement vers son aînée. Cet état de fait montre selon nous le « doubleusage » des dispositifs pour les jeunes filles qui viennent faire leurs devoirs (premier usage) et qui apprennent simultanément une partie du rôle de parents (deuxième usage). J'ai pu dénombrer pas moins de sept jeunes filles concernées par ce double-usage dans la population enquêtée ; ce qui correspond à la majorité des filles scolarisées en collège.

C'est pourquoi la situation des collégiennes nous a particulièrement interpellé dans cette recherche. Cet apprentissage des rôles parentaux qui arrive au moment où l'institution scolaire commence à questionner leur souhait d'orientation, semble être un vecteur d'hésitation. Les données récoltées ne permettent pas d'établir clairement un lien entre cet apprentissage et les choix d'orientation scolaire des jeunes filles si ce n'est le propos de Mathilde :

Comme travail je ne sais pas trop, comme je suis forte en dessin peut-être architecte ah mais non il faut être bonne en maths pour la géométrie et tout ça ou sinon médecin parce que j'adore la biologie et je suis trop forte en plus !

Interviewer : c'est des métiers intéressants.

Mathilde : Oui mais pour être médecin c'est long je crois...c'est 6 ans après le lycée non?!

Interviewer : 8 je crois...

Mathilde : tu vois c'est long et puis c'est dur y paraît ...et pour les enfants je sais pas comment ça se passe parce que c'est des horaires durs... 
thilde est la seule jeune fille à avoir exprimé aussi clairement ses réflexions par rapport à l'orientation en faisant inconsciemment un lien avec sa future vie de femme et de mère. Aucune ne nous a dit choisir un type d'études en fonction du temps qu'elle doit consacrer à sa fratrie. Néanmoins, l'exemple de Mathilde laisse penser qu'elles font un lien même inconscient entre les tâches qu'elles apprennent à effectuer auprès de leurs frères et sœurs, et le fait qu'elles devront les effectuer une fois mère et inscrites sur le marché du travail. Si nous n'avons pas plus d'éléments empiriques pour étayer ce point, l'apprentissage " préparateur » aux rôles parentaux que l'on vérifie chez la majorité des collégiennes nous invite à faire le lien avec F. Dubet (2010). Selon lui, les choix d'orientation paraissant conformistes relèvent en fait de la domination sociale et il n'est donc pas nécessaire que l'école discrimine pour que les individus finissent par s'autocensurer. C'est le cas de Mathilde qui se pose des questions sur son orientation et sans le savoir confirme les données du dernier rapport «Filles et garçons sur le chemin de l'égalité » (2010) montrant qu'à notes égales en mathématiques, les filles s'orientent moins facilement vers la filière $S$. Mathilde a pour son année de $4^{\text {ème }}, 14,5$ de moyenne en mathématiques, notation qui pourrait préserver son souhait de devenir architecte.

ll faut cependant garder à l'esprit que les individus ne sont pas entièrement assujettis aux déterminismes sociaux et que les filles peuvent avoir la « maîtrise » de leur orientation. Dans le dispositif $A$, Karine qui fréquente le dispositif depuis plusieurs années a, selon l'opinion des bénévoles et d'Olivia, pris de l'assurance et son assiduité l'a amenée à progresser rapidement. Je l'ai rencontrée quelques semaines avant qu'elle ne passe le baccalauréat, qu'elle a obtenu avec succès et qui lui a permis d'intégrer un IUT dans le domaine du commerce et de la gestion. Lors de nos échanges, elle m'a demandé des conseils pour le choix des établissements, comme elle n'hésitait pas à le faire sur tous les sujets d'ordre scolaire ou périscolaire avec l'ensemble des bénévoles. Elle étudie aujourd'hui à Lyon et projette de faire une année préparatoire aux concours d'entrée en HEC. Il y a donc aussi des exemples de jeunes filles fréquentant les dispositifs d'accompagnement qui « réussissent » à construire un projet d'études et de formation professionnelle selon leurs souhaits. Nous devons cependant indiquer que Karine n'avait pas la charge quotidienne de ses deux sœurs également lycéennes. Elle n'a pas eu à faire cet apprentissage du rôle parental que d'autres, comme Mathilde ou Tiphaine, effectuent.

\section{Conclusion}

Cette réflexion souhaite donc faire en sorte que l'on considère l'accompagnement scolaire comme un lieu où se jouent et se perpétuent également des apprentissages socio-sexués. Il ne s'agit pas uniquement d'y réaliser un travail scolaire mais aussi de répondre à un besoin social auprès des familles, qui pour solliciter les dispositifs d'accompagnement, sollicitent aussi les jeunes filles qui devraient se consacrer, comme leurs frères, à leur scolarité ou à leurs loisirs. C'est en cela que l'on peut observer un « double-usage » des dispositifs d'accompagnement. Le premier usage est relatif à la réalisation des devoirs. C'est donc un usage conscient et réfléchi, contrairement à l'usage socialisant qui concerne l'apprentissage des rôles parentaux par les jeunes filles. Nous n'avons pas abordé l'apprentissage de ces rôles par les garçons parce que nous n'en avons pas vu de traces. Est-ce à dire pour autant que l'accompagnement à la scolarité n'a pas d'influence sur eux à ce niveau là ? Il ne nous est pas possible de répondre scientifiquement à cette question pour le moment. L'hypothèse la plus vraisemblable que l'on puisse poser à ce sujet serait 
que les garçons faisant cet apprentissage plus tardivement que les filles pour diverses raisons, on ne peut pas en trouver de traces chez ceux qui fréquentent les dispositifs. Cet article n'aborde que certains angles de la question des inégalités filles-garçons dans le champ de l'accompagnement scolaire (aide à l'orientation scolaire, le parcours d'adhésion aux dispositifs, le rapport à la fratrie,etc.). Cependant, il ressort des résultats de l'enquête qu'un des apports des dispositifs d'accompagnement réside dans la qualité d'interface entre la famille et l'école d'une part, et les équipes de bénévoles et d'encadrant-e-s d'autre part. En effet, il semble que pour les jeunes, l'accompagnement à la scolarité soit entre autre chose, un endroit où ils et elles peuvent échanger sur leur orientation avec des personnes qui n'auront pas de plan préconçu pour eux. Il est perçu par les jeunes filles ayant participé à notre recherche comme un lieu leur permettant de faire leurs devoirs, mais aussi de discuter et d'échanger sur leur orientation avec des adultes. Ces personnes peuvent être une ressource pour les filles fréquentant les dispositifs, à condition d'être vigilant-e quant à leurs demandes mais aussi quant aux conditions de leur scolarisation. Dans cette optique, il est primordial que les partenaires éducatifs aient conscience des stéréotypes sexistes qu'ils et elles renvoient et ce, afin de mettre en place une éducation qui soit à la fois mixte et plus réellement égalitaire. La délégation de responsabilités parentales sur les filles doit être un élément à appréhender par les acteurs et actricesdes accompagnements, afin de veiller à ce qu'elles puissent vivre pleinement et sereinement la scolarité que mérite chaque élève quelque soit son identité de genre. Les jeunes filles soumises au double usage de l'accompagnement à la scolarité ne peuvent tout simplement pas avoir les mêmes chances de réussir leur scolarité et leur insertion professionnelle que les garçons car la société leur impose une charge supplémentaire, tout comme elle l'impose à la carrière de leurs mères, celle de prendre soin des autres avant de penser à elles.

\section{BIBLIOGRAPHIE}

Bacou, M. (2006). La mixité dans l'animation. Mixité, parité, genre dans les métiers de l'animation.

France : L'Harmattan.

Baudelot, C., \& Establet, R. (2008). Quoi de neuf chez les filles? Entre stéréotypes et libertés. France :

L'Harmattan.

Baudelot, C., \& Establet, R. (1992). Allez les filles! Une révolution silencieuse. Paris : Seuil.

Beaud, S., \& Weber, F. (2003). Guide de l'enquête de terrain. Paris : La découverte.

Belotti, E.-G. (1973). Du côté des petites filles. Paris : Des femmes Antoinette Fouque.

Bereni, L., Chauvin, S., Jaunait, A., \& Revillard, A. (2008), Introduction aux Gender Studies. Manuel des études sur le genre. Bruxelles : De Boeck.

Bonnéry, S. (2007). Comprendre l'échec scolaire. Elèves en difficultés et dispositifs pédagogiques. France : La dispute.

Dafflon Novelle, A. (Ed.). (2006). Filles-garçons : Socialisation différenciée ? France : PUG. 
Delalande, J. (2002). La cour de récréation. Pour une anthropologie de l'enfance. France : PUR.

Dubet, F. (2010). L'école « embarrassée » par la mixité. Revue Française de Pédagogie, 171, 77-86.

Duru-Bellat, M. (2008). La (re)production des rapports sociaux de sexe : quelle place pour l'institution scolaire ? Travail, Genre et Sociétés, 19, 131-149.

Duru-Bellat, M. \& Marin, B. (2010). La mixité scolaire, une thématique (encore) d'actualité ? Revue Française de Pédagogie, 171, 5-8.

Gillet, J.-C., \& Raibaud, Y. (Eds). (2006). Mixité, parité, genre dans les métiers de l'animation. France : L'Harmattan.

Glasman, D. (2001). L'accompagnement scolaire. Sociologie d'une marge de l'école. France : PUF.

Jaboin, Y. (2010). C'est bien... un homme à l'école maternelle !, Nouvelles questions féministes, 29(2).

Ministère de l'Education Nationale (2010). Filles et garçons sur le chemin de l'égalité, de l'école à

l'enseignement supérieur. France.

Ministère de l'Education Nationale, Ministère de l'Emploi et de la Solidarité, Ministère de la Jeunesse et des Sports, Ministère de la Ville, et le Ministère déléguée à la Famille, à l'Enfance et aux Personnes handicapées (2001). Guide de l'accompagnement à la scolarité. France.

Mosconi, N. (2003). Rapport au savoir et division socio-sexuée des savoirs à l'école, La lettre du grappe, 51, 31-38.

Safont-Mottay, C., Oubrayrie-Roussel, N., \& Prêteur, Y. (2010). Valeurs et attentes des collégiens et des collégiennes envers l'école : une mixité à construire. Revue Française de Pédagogie, 171, 31-45.

\section{RÉSUMÉS}

Cet article propose un regard sur l'égalité scolaire filles-garçons par le biais des dispositifs d'accompagnement à la scolarité en collectif, c'est-à-dire en équipements de quartier. Basé sur une recherche ethnographique, il s'interroge plus précisément sur la manière dont les enfants s'approprient les dispositifs d'accompagnement proposés. Les premiers résultats montrent qu'ils en ont un « double usage » de soutien scolaire et d'aide aux devoirs, mais aussi de socialisation et d'apprentissages des rôles parentaux, notamment par les filles. Les enfants sont «libres» de fréquenter l'un ou l'autre des dispositifs du quartier. Cette « liberté » s'exerce dans une logique d'appropriation sexuée du quartier comme territoire de jeux et de socialisation qui entre en correspondance avec les spécialisations de l'offre d'accompagnement scolaire locale. Cette correspondance n'est pas sans rappeler les rapports socio-sexués au savoir, qui sont construits au sein de l'école. Ainsi, on constate d'une part que l'appropriation est genrée et d'autre part qu'elle reflète les scolarités proposées aux filles et aux garçons. En ne tenant pas compte de la socialisation sexuée et des rapports socio-sexués au savoir, les dispositifs vont dans le sens des inégalités de genre en construction chez les jeunes.

This article looks at gender inequality in specific help projects for students. In French city suburbs, children are offered to get some extra homework help. These projects are implemented by associations in order to help students who need to be explained how to learn and do their homework. Based on an ethnographic approach, this research tried to determine how children appropriate themselves this help. First results indicate that children have a "dual use" of homework help projects. The first use is about homework, but there is another use for girls who have to learn parental duties, such as taking care of their brothers and sisters so that boys don't have to worry about that. Children are also free to attend one or the other of the projects usually 
implemented in their districts, and the data show that they see this help as any other playgrounds in their environment. However, the way the children disseminate in the projects fits the different specializations of the projects. Boys are actually attending mathematics projects and girls' literature projects. So we can say that children have a gendered use of homework help projects, that reflects their education in our society.

INDEX

Mots-clés : dispositif d'accompagnement à la scolarité, rapport au savoir, rôle parental, socialisation sexuée

Keywords : gendered socialization, parental duties, relation to knowledge, specific help project for student

\section{AUTEUR}

\section{CYRILLE MORIN}

Attaché Temporaire d'Enseignement et de Recherche - Université de Rennes 2 Haute Bretagne /Université Européenne de Bretagne. 